\title{
Zur Stellung der Geographie in der Umweltforschung
}

Ernst Winkler
Die große Umwelt formt uns, die kleine Umwelt formen wir.
Der unmittelbare Anlaß zu den folgenden Erörterungen war die beunruhigende gegenwärtige Situation unseres Lebensraumes, die in Schlagworten wie «Umweltgefährdung», «Umweltverbrauch» und «-mißbrauch» sowie «Umweltzerstörung» einerund "Umweltschutz» oder «Umweltregeneration» andrerseits zum Ausdruck kommt. Mittelbar drängten zur Niederschrift die ständig häufiger werdenden Forderungen nach entsprechenden Aktionen und Institutionen (Forschungsinstituten usw.), die zudem je länger desto gebieterischer erhoben werden. Sie geben zu erkennen, daß mit seiner Lebenssphäre auch die Existenz des Menschen selbst in Schwierigkeiten geraten ist. "In dem Maße», sagte Präsident R. Nixon am 9. Juli 1970 in einer an den Kongreß der USA gerichteten Sonderbotschaft, "in dem die Besorgnis über den $\mathrm{Zu}$ stand unserer natürlichen Umwelt wächst, wird auch immer deutlicher, daß wir mehr über die Umwelt in ihrer Gesamtheit wissen müssen. Es wird immer deutlicher, daß wir nur durch eine Reorganisation der zuständigen... Behörden dieses Wissen schaffen und den Schutz, die Entwicklung und die Verbesserung der gesamten Umwelt wirksam sicherstellen können»1. Solche Worte, die kaum als bloße rhetorische Politika gelten können, und die Erklärung des Jahres 1970 zum Europäischen Naturschutzjahr erhärten die Tatsache, daß dieser Schutz zum dringlichen weltweiten Anliegen geworden ist².

Damit ist selbstverständlich - wie bereits angedeutet - zugleich die gesamte Forschung und mit ihr die Geographie, die man nicht selten mit Umweltwissenschaft identifiziert, aufgerufen (wobei gesagt werden kann, daß im Grunde alle Wissenschaft Umweltforschung ist). Die Geographie hat deshalb besonders aufzuhorchen, wenn Manifestationen für die Umwelt veranstaltet werden. Könnte doch aus ihnen für sie abgeleitet werden, sie habe sich bisher offenbar zu wenig um diese Umwelt gekümmert (was auch die Entstehung neuer [Ersatz-]Disziplinen: Ökologie, Raum- und Regionalforschung, die Sozialökologie im speziellen zu belegen scheint). Auf der andern Seite ließe sich ebensowohl folgern, daß die Geographie noch notwendiger geworden sei als früher und ihre Anstrengungen zur Erkenntnis der (landschaftlichen) Umwelt zu steigern habe. Für beide Fälle ist die Frage zu prüfen, welches das Verhältnis der Geographie zur Umweltforschung sei und welche eventuellen Möglichkeiten bestehen, es zugunsten einer optimalen Umweltpflege auszubauen. Hiefür bedarf es zunächst wohl einer kur- zen Diskussion der Begriffe «Umwelt» und «Umweltforschung». Nehmen wir den erstgenannten vorweg, da er Anlaß unserer Erörterungen bildet, so mag es vielen müßig erscheinen, ihn überhaupt in Frage zu ziehen. Denn «man weiß selbstverständlich", was unter Umwelt zu verstehen ist. In Tat und Wahrheit besitzt dieses Wort so viele Bedeutung, daß es schwer hält, dafür einen gemeinsamen «Nenner» zu finden. Dies belegt schon ein flüchtiges Streiflicht auf seine Geschichte. Nach Trübners Wörterbuch der deutschen Sprache geht das Wort auf den dänisch-deutschen Dichter J. Baggesen zurück, der es um 1800 in einer Ode verwendete $^{3}$. Darin bedeutete es mutmaßlich vor allem (Nah-)Umgebung (des Menschen), wobei nicht klar wird, ob Baggesen es auch schon in weiterem Sinn verstand. In der Folge behielt es zwar grundsätzlich diesen Sinn im wesentlichen bei, doch weiteten ihn einzelne Wissenschaften wie etwa die Psychologie und die Philosophie in Richtung "Außenwelt» oder «Welt» überhaupt. Der Biologe J. v. Uexküll, einer der bedeutendsten Begründer moderner Umweltlehre schließlich, stellte empirisch fest, daß jedes Lebewesen eine «eigene» Umwelt besitzt4.

Vom Proton (Elementarpartikel) bis zum Weltall gibt es also "Myriaden» verschiedener Umwelten. Gewissen Forschern mag dies ein Grund zur Forderung sein, den Terminus "Umwelt» aus der Wissenschaft zu verbannen - in Analogie etwa zum Landschaftsbegriff, der vielen Geographen einen Stein des Anstoßes darstellt. Das sinnvollere Verfahren dürfte indessen sein, den offenbar "fruchtbaren" Begriff klar zu fassen und ihn dann objektgemäß zu spezifizieren. Wie der Biologe K. Friederichs nachwies, gilt es dabei zu beachten, $\mathrm{da} ß$ «Umwelt» ein Stufenbegriff ist ${ }^{5}$. Friederichs selbst gliederte ihn in sechs Hauptstufen: 1. als "Weltzusammenhang» in bezug auf ein Lebewesen; 2. als Komplex von Außenfaktoren, mit denen die Lebewesen in direkter oder konkret greifbarer indirekter Beziehung stehen; 3. als Komplex der lebenswichtigen Beziehungen, die in Wirkung und Gegenwirkung das $\mathrm{Ma}$ der Entfaltung des Lebens einer Art bestimmen; 4. als Komplex der direkten Beziehungen eines Lebewesens zur Außenwelt (als) "physiologische Umwelt»); 5. als "Minimalumwelt», das heißt als Komplex der für die Art lebensnotwendigen Außenfaktoren und endlich 6 . als "vorwiegend psychologischen Umweltbegriff» (nach J. v. Uexküll). Diese deutlich subjektbezogene Gliederung läßt sich auch objektivieren, indem z.'B. kosmische, planetare, 
terrestrische und teilterrestrische (regionale) Umwelten unterschieden werden können. Damit ergibt sich die Möglichkeit einer durchaus klaren Systematisierung, die beispielsweise in der Biologie (Ökologie) grundsätzlich bereits besteht. $\mathrm{Da} \beta$ sich die einzelnen Umwelten überschneiden und überlagern, daß sie meist ohne scharfe Grenzen ineinander übergehen und überdies Bestandstücke einer Gesamtumwelt, des Weltalls, darstellen, kann kaum als Hemmnis der Forschung betrachtet werden. Gilt - mindestens funktionell - der im vorhergehenden Satz angenommene Zusammenhang doch für sämtliche Objekte aller Wissenschaften. Das Gefüge der Umwelten mutet insofern sogar noch konkreter an als dasjenige der "Sachobjekte», da diese entscheidend von jenen (und der Gesamtumwelt) bestimmt werden. Erst ein System von Dingen und ihren Umwelten darf mit andern Worten volle Realität beanspruchen. Doch liegt dieses Thema außerhalb unserer Erörterung.

Um auf diese zurückzublenden, läßt sich festhalten, $\mathrm{da}$ dem skizzierten System der Umwelten einleuchtenderweise ein solches der Umwelt-Wissenschaften entspricht, wiewohl es tatsächlich erst sehr rudimentär existiert. Einer Kosmologie oder einer Gruppe von Kosmologien (Kosmophysik, -chemie, -biologie usw.) als Gesamtumweltlehre(n) sind so unter anderem die Erdwissenschaften subsumierbar, Disziplinen also, welche sich die Erde oder Teile von ihr zum Gegenstand erwählt haben. Sie sind in die Teildisziplinen etwa der Atmosphären-, Hydrosphären-, Lithosphären- und Biosphärenforschung unterzugliedern, von denen aus noch weiter zu den Organismen- und Anorganismenwissenschaften fortgeschritten werden kann. Für sie alle funktioniert der Forschungszweig, der sich dem Komplex oder dem Gefüge von Litho-, Atmo-, Hydro- und Biosphären, der Landschaftssphäre (auch Geo- oder Chorosphäre genannt) widmet, die Geographie oder Landschaftsforschung als eine Art «Integrationszentrum».

Damit ist deren Verhältnis zu den (übrigen) Umweltlehren bzw. zur Umweltforschung überhaupt umrissen. Es läßt sich indessen präzisieren. Hierzu wird zweckmäßigerweise nochmals auf die Gegenstände bzw. Betrachtungs- oder Forschungsrichtungen dieser Disziplinen zurückgegriffen. Setzen wir Umweltlehren gleich Ökologien, da jene meist nicht nur als solche angesprochen, sondern definiert werden, dann darf gesagt werden, daß in ihrem Blickzentrum immer ein Lebewesen oder eine Organismengemeinschaft (Gesellschaft, Vergesellschaftung) steht, wobei freilich Umweltlehren der «Anorganismen" nichts im Wege liegt ${ }^{6}$. In vielen, wenn nicht den meisten Umweltdiskussionen der Gegenwart wird sogar nur an letztere, also an die Welt der Gewässer und an die Luft (inkl. Lärm) gedacht. Die Betrachtungsrichtung dieser Ökologien ist die von der Umwelt zum Organismus; die Grundfrage zielt nach den Wirkungen der Umwelt auf die Lebewesen. Dabei werden anorganische und organische (beim Menschen auch kulturelle oder soziale) Umwelten unterschieden. Das Visier der Geographie als Landschaftssphärenwissenschaft (auch LandschaftsSinne umgekehrt - von den Organismen und Anorganismen auf die Umwelt selbst, genauer, auf eine bestimmte, die landschaftliche Umwelt oder einfach die Landschaftssphäre. In dieser bilden die Lithosphäre, Atmosphäre, Hydrosphäre und Biosphäre die Teile oder Faktoren (also das, was die Umwelt für den Ökologen bedeutet).

Damit ist ausgedrückt, daß die Geographie als Landschaftssphärenwissenschaft (auch Landschaftssphärologie) sich keineswegs mit Umweltforschung schlechthin identifizieren läßt. Sie hat sich bewußt als eine unter andern Umweltdisziplinen zu sehen. Allerdings ist ihr, da die Landschaftssphäre als Synthese bestimmter terrestrischer (Hüllen-)Sphären (Litho-, Atmo-, Hydro- und Biosphäre) in gewissem Sinne eine Schlüsselposition im Leben des Menschen innehat (weil dieses wie übrigens alles Leben nicht von einzelnen dieser Sphären, sondern von ihrer Gesamtheit bestimmt wird), eine besondere Aufgabe zugeordnet. Sie ist vor allem darin zu erblicken, bei der Beurteilung der Wirkungen der landschaftlichen Umwelt auf den Menschen eine Überbetonung einzelner (Teil-)Sphären zu verhindern, positiv gewendet, diese in ihrer wahren quantitativ-qualitativ-realen Anteilhaftigkeit im Ganzen (der Landschaft) zu erfassen, was von anderer Seite nicht selten vernachlässigt wird. Die genannte Aufgabe bringt übrigens die grundlegende Funktion der Geographie für alle Landschaftspraxis, für Landschafts- und Landesplanung, -gestaltung, -pflege, -schutz und -nutzung zum Ausdruck, die optimal nur mittels objektgemäßer Aufeinanderabstimmung sämtlicher Interessen möglich ist.

Mit dieser Feststellung will keineswegs gesagt sein, $\mathrm{da}$ der Landschaftsforschung größere Bedeutung als andern Wissenschaften zukommt. Es handelt sich mit andern Worten beim Vergleich der Geographie mit den übrigen, insbesondere Geo-Disziplinen, nicht um eine Qualitätsdifferenz, sondern um eine Frage verschiedener Ebenen bzw. Komplexitätsgrade der Objekte, und die Geographie selbst ist sich klar bewußt, daß auch sie wie alle übrigen Glied einer Kette darstellt. Andrerseits darf sie ebenso bestimmt betonen, daß sie so wenig überflüssig ist wie sämtliche übrigen Umweltwissenschaften, und dies unbeschadet der Tatsache, ob sie ihre Probleme hinreichend löst oder nicht. Man könnte sogar ganz allgemein behaupten, daß ein solcher die Erkenntnis des Ganzen der Landschaft erstrebender Wissenszweig, falls er nicht bestünde, gerade im Blick auf die Existenz des Menschen notwendig zu schaffen wäre. 
Zusammenfassend darf gefolgert werden: Die Landschaftsforschung oder Geographie ist primär Erdwissenschaft, deren Standort innerhalb der übrigen Einzelwissenschaften von der Erde und auch der Umweltdisziplinen durch den Kontakt der Litho-, Atmo-, Hydro- und Biosphäre fixiert wird. Das aus diesem Kontakt resultierende (höhere) Objektgefüge, die Landschaftssphäre, bildet einen besonderen Erkenntnisgegenstand, der für den Menschen eine speziell existenzwichtige Form der Umwelt repräsentiert, für die und ihre einzelnen Arten übrigens im Wort «Ökosystem» (von A. G. Tansley, 1935) ein gebräuchlicher Fachausdruck besteht.

Hieraus läßt sich noch eine nicht unwichtige weitere Folgerung ableiten. Als Landschafts(sphären)forschung ist die Geographie ausschließlich Theorie. Ihren "Sinn» empfängt sie indessen - wie jede Wissenschaft - allein aus der Anwendbarkeit ihrer Erkenntnisse. Die Anwendbarkeit wiederum ist gegeben durch Landschafts- oder Landesplanung, -gestaltung, -pflege (inkl. -schutz und -bau) sowie Landschafts- und Landesnutzung. Von der Leistung für diese Tätigkeitsbereiche wird die Existenz der Landschaftsforschung oder Geographie weitgehend abhängen, womit die Erörterung in die Eingangsthemen zurückmündet. Zu hoffen bleibt hierbei, daß bei den kommenden weiteren Diskussionen über Umweltschutz und Umweltpflege auch der Landschaftsforschung gebührend gedacht wird, wobei sie selbst allen Grund hat, sich energisch und mit positiven Beiträgen einzuschalten. Für die Landschaftstheorie wie für alle Landschaftspraxis vermag das Kants Imperativ in die "Umweltsprache» übersetzende Wort des - bereits wiederholt erwähnten Biologen J.v. Uexküll die allgemeine und unbestreitbare Richtlinie zu bieten: Baue deine Umwelt so, daß sie allen anderen Umwelten als Vorbild dienen kann.

\section{Hauptsächlich benützte Quellen}

1 Sonderbotschaft Präsident Nixons über die Schaffung eines Bundesamtes für Umweltschutz und einer Abteilung für Fragen der Luftreinhaltung und der Meeresnutzung im Handelsministerium. United States Information Services. Bad Godesberg 1970. Vgl. auch Botschaft Präsident Nixons zur Umwelthygiene vom 10. Februar 1970. (Deutsche Übersetzung. Manuskript.) Schon 1961 hatte übrigens J. F. Kennedy analoge Maßnahmen gefordert.

2 Hunziker, Th.: Die Zielsetzung des europäischen Naturschutzjahres 1970. Schweiz. Ztschr. f. Forstwesen $6,121,1970$.

3 Trübners Deutsches Wörterbuch. Bd. 7. Berlin 1956.

4 v. Uexküll, J.: Umwelt und Innenwelt der Tiere. Berlin 1921; derselbe: Theoretische Biologie. 2.
Aufl. Berlin 1928; ders.: Streifzüge durch die Umwelten von Tieren und Menschen. Bedeutungslehre. Hamburg 1958.

5 Friederichs, K.: Umwelt als Stufenbegriff und als Wirklichkeit. Studium Generale 2/3, 3, 1950. - Vgl. dazu: Plessner, H.: Über das Welt-Umweltverhältnis beim Menschen. Daselbst - Mühlmann, W. E.: Das Problem der Umwelt beim Menschen. Ztschr. für Morphologie und Anthropologie 2, 44, 1952. Scheler, M.: Die Stellung des Menschen im Kosmos. München 1947. - Gombert, A.: Umwelt. Ztschr. f. deutsche Wortforschung 2, 7, 1905. - Schmidt, P. H.: Das Ich und die Umwelt, Individuum und Gemeinschaft. Festschr. zur Fünfzigjahrfeier der Handelshochschule St. Gallen 1949. - Barrows, H. H.: Geography as Human Ecology. Annals of the Assoc. of American Geographers 3, 12, 1923. - Eyre, S. R. und Jones, G. R. J.: Geography as Human Geography. London 1966. - Tulippe, O.: Initiation à la géographie humaine. Liège 1949. - Dahmen, F.W.: Ansatzpunkte zur Lösung des Umweltproblems. Garten und Landschaft H. 6, 1970. - Nestmann, L.: Die Humanökologie - Begriff, Inhalt und Stellung im System der Wissenschaften. Deutsche Universitätszeitung H. 5, 1968.

6 So faßte z. B. J. Tricart in "Qu'est-ce que la Géomorphologie?» diese Disziplin (Revue générale des Sciences, H. 2, 57, 1950) als Ökologie auf. Vgl. Geographica Helvetica H. 2, VI, 1951. - Castaldi, F.: Geografia ed Ecologia. Atti del XVII Congresso Geogr. Italiano, Bd. III, Bari 1957.

Für die gesamte Frage: Drdos, J.: Über die Betrachtung der Landschaft als System in der Landschaftsökologie. Hannover 1970. - Langer, H.: Wesen und Aufgaben der Landschaftsökologie. Hannover 1968. - Ders. : Landschaftspflege als Raumplanung, Natur und Landschaft 1, 45, 1970. Meyer-Abich, A.: Geistesgeschichtliche Grundlagen der Biologie. Stuttgart 1963. - Cohen, S. B. (Herausgeber): Geography and the American Environment. O. O. 1968. - Tienemann, A. F.: Leben und Umwelt, Hamburg 1956. - Harvey, D.: Explanation in Geography. London 1969. - Fuchs, G.: Der Wandel zum anthropogeographischen Denken in der amerikanischen Geographie. Marburg 1966. - Gerasimov, I. P.: Die Wissenschaft von der Biosphäre und ihrer Umgestaltung. Petermanns Geogr. Mitteilungen 1, 113, 1969. - Wasmer, H. R.: Umwelthygiene im Weltraum. Jb. Chemische Industrie 1969 (Sonderdruck, 2 S.). - Jungk, R. und Calder, N.: Vor uns das Paradies, London, München 1968. - Pulyarkin, V. A.: On the Content of the Concept "Geographical Environment" and the Influence of the Environment in Society Soviet Geography 10, 1969, No. 5 - Yefremov, Y. K.: The Landscape Sphere and the Geographical Environment. Soviet Geography 10, 1969, No. 5. 